\title{
Metatranscriptomics reveals metabolic adaptation and induction of virulence factors by Haemophilus parasuis during lung infection

\author{
Bernardo Bello-Ortí ${ }^{1}$, Kate J. Howell², Alexander W. Tucker², Duncan J. Maskell ${ }^{2}$ and Virginia Aragon ${ }^{1 *}$
}

\begin{abstract}
Haemophilus parasuis is a common inhabitant of the upper respiratory tract of pigs, and the causative agent of Glässer's disease. This disease is characterized by polyserositis and arthritis, produced by the severe inflammation caused by the systemic spread of the bacterium. After an initial colonization of the upper respiratory tract, $H$. parasuis enters the lung during the early stages of pig infection. In order to study gene expression at this location, we sequenced the ex vivo and in vivo $H$. parasuis Nagasaki transcriptome in the lung using a metatranscriptomic approach. Comparison of gene expression under these conditions with that found in conventional plate culture showed generally reduced expression of genes associated with anabolic and catabolic pathways, coupled with up-regulation of membrane-related genes involved in carbon acquisition, iron binding and pathogenesis. Some of the up-regulated membrane genes, including $A B C$ transporters, virulence-associated autotransporters (vtaAs) and several hypothetical proteins, were only present in virulent $H$. parasuis strains, highlighting their significance as markers of disease potential. Finally, the analysis also revealed the presence of numerous antisense transcripts with possible roles in gene regulation. In summary, this data sheds some light on the scarcely studied in vivo transcriptome of $\mathrm{H}$. parasuis, revealing nutritional virulence as an adaptive strategy for host survival, besides induction of classical virulence factors.
\end{abstract}

\section{Introduction}

Haemophilus parasuis is the causative agent of Glässer's disease, an infectious disease of pigs characterised by fibrinous polyserositis. Current strategies for disease control are based on rapid diagnostics, the use of antibiotics and to a lesser extent vaccines [1]. Antibiotics have been extensively used for this purpose, but current recommendations focus on reduction of their use to avoid the emergence of drug resistance [2-4]. Antibodies can control disease [5] in a mechanism that, at least in part, relies on opsonisation, which renders the virulent phagocytosis resistant strains susceptible to killing by alveolar macrophages [6]. Vaccines, as well as probiotics, are candidates to replace antimicrobials as preventive agents $[7,8]$. Virulence factors, especially those important for the initial stages of infection, are ideal targets for vaccine

\footnotetext{
* Correspondence: virginia.aragon@irta.es

'Centre de Recerca en Sanitat Animal (CReSA), Institut de Recerca i Tecnologia Agroalimentàries (IRTA), Campus de la Universitat Autònoma de Barcelona, Bellaterra, 08193 Cerdanyola del Vallès, Spain

Full list of author information is available at the end of the article
}

design in order to block the pathogenesis potential of bacteria. In that regard, some $H$. parasuis virulence factors have been reported in the literature, and were reviewed recently $[9,10]$.

Numerous works have indirectly linked specific $H$. parasuis genes to its pathogenicity, but direct demonstration of their role during infection is still lacking. In addition, these studies have been typically driven by the homology to previously reported virulence factors in other bacterial species from the Pasteurellaceae family. Moreover, pathogenic mechanisms, such as immunomodulation or mechanisms for nutrient acquisition during host infection, could be linked to unsuspected virulence factors [11,12]. After intranasal inoculation, virulent $H$. parasuis can be detected in the lung, from where it can spread causing systemic infection, with the consequent severe inflammation $[13,14]$. In the lung, $H$. parasuis is detected inside macrophages and neutrophils, but also within epithelial cells [14]. Survival of $H$. parasuis in the lung environment seems to be linked to phagocytosis 
resistance capacity of the strain, but other unknown virulence mechanisms cannot be ruled out $[14,15]$. To address this issue, in vivo approaches coupled with hypothesis generating strategies, such as high-throughput RNA sequencing (RNA-seq), could add additional insight into $H$. parasuis pathogenic mechanisms. To our knowledge, no studies have been reported regarding transcriptomic analysis of $H$. parasuis during infection. Few papers have been published in the Pasteurellaceae family, but only Jorth et al. [16] applied high-resolution transcriptomics [16-19]. To fill this gap in $H$. parasuis infection control, we used a metatranscriptomic approach to study $H$. parasuis pathogenesis in the pig lung. Gene expression profiling, and more recently RNA-seq, has been established as the de facto gold standard technique to tackle the survival strategies of numerous bacterial pathogens [20-22]. The specific objective of this work was to study $H$. parasuis gene expression during lung infection, with a special focus on previously reported virulence factors [10]. We found that $H$. parasuis changes its global gene expression during lung infection. A down-regulation of $H$. parasuis metabolism in the lung was accompanied by the induction of the expression of known virulence-factors together with genes of unknown function.

\section{Materials and methods}

\section{RNA samples and sequencing}

The virulent $H$. parasuis Nagasaki strain was chosen for transcriptomic analysis [GenBank: ANKT01000000]. This strain was originally isolated from the meninges of a pig with a systemic infection by $H$. parasuis in Japan. Gene annotations are based on previous analysis [23]. Further pathway inspection was performed with Integrated Microbial Genomes (IMG) [24] and BioCyc [25].

Animal experiments were performed in accordance with the regulations required by the Ethics Commission in Animal Experimentation of the Generalitat de Catalunya (Approved Protocol number 5796).

To examine gene expression during lung infection, ex vivo incubation of the bacteria in porcine lungs was carried out. Nagasaki grown overnight on chocolate agar plates was resuspended in a final volume of $20 \mathrm{~mL}$ sterile PBS (aprox. $10^{7} \mathrm{CFU} / \mathrm{mL}$ ). A healthy 6 week old pig was euthanised and lungs were extracted under sterile conditions and transported to the laboratory, where they were inoculated with the bacterial suspension. After $2 \mathrm{~h}$ of incubation at $37{ }^{\circ} \mathrm{C}$ with $5 \% \mathrm{CO}_{2}$, bacteria were recovered from the lung by lavage with $100 \mathrm{~mL}$ of cold sterile PBS. Colony counts were performed before and after incubation. Next, the brochoalveolar lavage fluid (BALF) was subjected to differential centrifugation to eliminate pulmonary cells $(460 \times g, 10 \mathrm{~min})$ and recover bacteria in a second centrifugation $(3220 \times g, 20 \mathrm{~min})$. The bacterial pellet was processed for RNA extraction following two previously described hot phenol extractions [26]. The protocol included depletion of host and bacterial rRNA with a Ribo-Zero kit (Epicentre). This bacterial RNA sample is referred to as the ex vivo sample for simplicity through the rest of the text. A sequencing library was generated using an Ion Torrent RNA-Seq v2 kit (Life Technologies) and RNA was sequenced using an Ion Torrent PGM instrument (Life Technologies) with an Ion 316 chip (Life technologies) at the Centre for Research in Agricultural Genomics (CRAG, Campus de Bellaterra-UAB, Spain). RNA from the Nagasaki strain grown overnight on chocolate agar plates was purified and sequenced in the same manner and served as the control. A replicate of the complete experiment was processed in the same manner.

To assess the validity of the ex vivo model, RNA was obtained from Nagasaki recovered from the lungs of a pig after a short in vivo infection. Nagasaki grown overnight at $37{ }^{\circ} \mathrm{C}$ and $5 \% \mathrm{CO}_{2}$ on chocolate agar plates was resuspended in $20 \mathrm{~mL}$ of PBS (aprox. $10^{7} \mathrm{CFU} / \mathrm{mL}$ ). This bacterial suspension was intratracheally inoculated in a 6 week old pig. A second pig was mock inoculated with the same quantity of PBS. After two hours, the two pigs were euthanized and lungs were processed as above to obtain a bacterial pellet. This bacterial sample is referred to as the in vivo sample for simplicity through the rest of the text. The pellets from the low speed centrifugation containing alveolar macrophages were processed to study the expression of surface markers CD163, sialoadhesin (or CD169), SLAI and SLAII by flow cytometry following a previously described protocol [27]. Bacterial RNA was purified as above and libraries were generated using NEBNext ${ }^{\circ}$ Ultra $^{\text {Tx }}$ Directional RNA Library Prep Kit for Illumina ${ }^{\circ}$. Sequencing in one paired-end Illumina MiSeq run was performed at the Institut de Biotecnologia i Biomedicina (IBB, Campus de Bellaterra-UAB, Spain). RNA from the Nagasaki strain grown overnight on chocolate agar plates was purified and sequenced in the same manner and served as the control. The NEBNext Ultra Directional kit uses the dUTP method to preserve "directionality of the library".

\section{Transcriptomic analysis}

RNA-seq analysis was based on the $H$. parasuis Nagasaki draft genome. The genome is $2.3 \mathrm{Mb}$ in length and was assembled in 47 scaffolds [23]. The Nagasaki genome encodes 2260 protein-coding genes (with 40 additional putative pseudogenes): 329 annotated as transporters, 754 connected to KEGG pathways and 1418 to KEGG orthology (KO), 1571 are COG annotated and 1918 associated to Pfam. This information is available in IMG.

For both in vivo and ex vivo experiments, bioinformatic analysis was performed following the count-based differential expression method [28], with some modifications 
[26]. The procedure was the same as that used previously; however, BWA aligner v0.7.9a-r786 was used [29] and minimum mapping quality (MAPQ) was increased to 20 . SAMStat was used to get additional mapping statistics [30]. Only protein coding genes with one or more counts per million (cpm) in at least one of the samples compared were used. EdgeR tool was chosen for differential gene expression, defining differentially expressed genes at $F D R<0.05$. EdgeR biological coefficient of variation $(\mathrm{BCV})$ was calculated using the data from the ex vivo experiment, and was also used to analyse the in vivo data. Enrichments were performed using the BLAST2GO built-in tool [31], using $P<0.05$ as the threshold. Enrichment results were further processed using the REVIGO online tool [32].

In an attempt to detect putative virulence factors, up-regulated genes in the in vivo or ex vivo experiments compared to agar plate growth were further analysed. Transmembrane domains and the presence of signal peptide motifs were predicted using Phobius [33]. In addition, the presence of these membrane-related genes in a database containing 10 virulent and 14 non-virulent isolates of $H$. parasuis was also studied. This database contains some of the $H$. parasuis genomes that were recently published [34] [EMBL:ERS132054- EMBL:ERS132066, EMBL:ERS132069, EMBL:ERS132073, EMBL:ERS132075, EMBL:ERS132076, EMBL:ERS132078- EMBL:ERS132084]. Automated genome annotation was performed by Howell et al. [34]. The predicted proteome of Nagasaki was compared against the database of $H$. parasuis proteins using BLASTP with a cut-off value of $E=1 \times 10-5$. Proteomes were parsed from GenBank files using a Biopython script. The settings to define homology were the following: identities $\geq 90 \%$ and query coverage $\geq 80 \%$ per highscoring segment pair (HSP). All transcriptomic data were deposited in the Gene Expression Omnibus database [GEO: GSE63851].

Non-coding RNA (ncRNA) were also examined. Read mapping to the complement strand were analysed with the same pipeline as the sense reads, including differential expression and enrichment. Antisense RNA (asRNAs) have been reported in the literature overlapping more than one gene, but also partially overlapping the target gene, commonly known as cis-encoded sRNA. For simplicity, in this study we took one gene as the unit of analysis, and therefore we did not take into account the possible overlap of an asRNA with more than one gene. Reverse transcription (RT)-PCR was performed to validate four asRNA using SuperScriptTM III Reverse Transcriptase (Life Technologies). Only a forward primer was used in the RT step. Each putative asRNA was validated in plate and in vivo RNA samples, using Nagasaki DNA as a positive control. To ensure DNA absence, samples without prior reverse transcription were also amplified.

\section{RT-qPCR}

To validate in vivo and ex vivo RNA-seq runs, primers were designed for selected genes (listed in Additional file 1). Also, some putative virulence factors were validated in these two samples, as well as in additional in vivo samples from a previous experiment [27]. Briefly, snatch-farrowed colostrum-deprived piglets were intranasally inoculated with $5 \times 10^{6} \mathrm{CFU}$ of Nagasaki. After 1, 2 and 3 days post infection (dpi), pigs were euthanized and lungs were recovered. Bronchoalveolar lavage was performed and bacteria were obtained for RNA purification as described above. Samples were retrotranscribed using SuperScript ${ }^{\circ}$ VILO $^{\text {in }}$ Master Mix (Life Technologies). qPCR were performed in triplicates in the 7500 Fast Real-Time PCR System with SYBR $^{\circ}$ Green Real-Time PCR Master Mix (Life Technologies). Each sample belonged to one animal at the specific time post infection. Expression of the selected genes was compared to plate culture. The results were analysed using the $\Delta \Delta C_{t}$ method.

\section{Results}

As a facultative anaerobe, $H$. parasuis Nagasaki is expected to have the ability to generate energy via fermentation or respiration. In fact, when metabolic pathways were inspected using IMG and BioCyc databases, genes involved in both aerobic and anaerobic respiration were detected (oxygen and nitrate as terminal electron acceptors), as well as formate and acetate fermentation genes. Sugar transport systems such as ATP-binding cassette (ABC) transport complexes and phosphotransferase systems (PTS) were encoded in its genome, as well as the genes associated to glycolysis, gluconeogenesis, tricarboxylic acid (TCA) cycle and the pentose phosphate pathway. From the anabolic side, pathways for the biosynthesis of amino acids, nucleotides, cofactors, fatty acids as well as heme were present. Natural competence, adherence and secretion capabilities, lipooligosaccharide (LOS) biosynthesis, proteases and iron acquisition completed the repertoire of genes that should give the Nagasaki strain the potential to colonize and survive during host infection.

In order to mine RNA-seq results, a custom GO term database was built to include more functionally annotated genes and therefore be potentially more suitable for pathway mining than the KEGG annotations assigned by IMG. Using this custom database, at least one GO term was assigned to $74 \%$ of the 2260 protein coding genes. A good GO level distribution (mean level $=6.8 ; \mathrm{SD}=2.7$ ) and more than 8000 annotations were used for pathway mining. Since $25 \%$ of the genes did not have any associated GO term, they were not taken into account when performing enrichments, and a separate analysis had to be performed to mine possible up-regulated membranerelated genes. 


\section{RNA-seq run statistics}

A range of 2.8-3.2 $\mathrm{M}$ and 2.6-3.7 $\mathrm{M}$ reads were obtained with Ion Torrent from the ex vivo samples and the corresponding plate samples, respectively. Alignment to the Nagasaki genome was successful for $53-87 \%$ of the reads from the ex vivo samples and $61-90 \%$ of the reads from the pure plate cultures. To perform differential expression, BCV was calculated and a value of 0.3 was obtained. Differential expression analysis of the ex vivo samples revealed 765 differentially expressed genes (DEG) with statistical significance $(F D R<0.05)$. From these, 393 were up-regulated and 372 down-regulated after $2 \mathrm{~h}$ incubation in lung explants (Figure 1A and Additional file 2A). RNAseq results were validated by RT-qPCR using selected genes belonging to key altered pathways, such as cofactor biosynthesis (6-pyruvoyl tetrahydrobiopterin synthase), TCA cycle (type II citrate synthase), phenylalanine, tyrosine and tryptophan biosynthesis (anthranilate synthase component II), heme export (heme exporter protein D), mannose transport (PTS system mannose-specific transporter subunit IID) and nutrient transport (ABC transporter inner membrane subunit), with the latter also being a putative virulence factor (Table 1). Differential gene expression was confirmed with a significant correlation between RT-qPCR and RNA-seq $(r=0.95, P<0.05)$.

For the in vivo experiment, high-resolution Illumina sequencing was chosen. A total of $18 \mathrm{M}$ and $15 \mathrm{M}$ reads were obtained for the in vivo sample and the corresponding plate sample, respectively. From the total, 15\% and $83 \%$ mapped to the Nagasaki genome, for in vivo and plate culture, respectively. This $15 \%$ accounted for 2.7 $\mathrm{M}$ reads that mapped to the Nagasaki genome from the total in vivo reads. To perform differential expression, a BCV of 0.3 was used, as obtained with the ex vivo data. Differential expression analysis between both samples yielded 542 DEG: 369 were up-regulated and 173 down-regulated after $2 \mathrm{~h}$ pig infection (Figure $1 \mathrm{~B}$ and Additional file 2B). The differential expression was validated by RT-qPCR with the same genes used for the ex vivo experiment (Table 1). The correlation between the results by RT-qPCR and RNAseq was lower in this case but still statistically significant $(r=0.8, P<0.05)$.

\section{Differential gene expression and enrichment}

Gene set enrichments for housekeeping, up-regulated and down-regulated genes were performed in order to have an overview of $H$. parasuis transcriptomic response in the lung. With regards to the ex vivo incubation, analysis revealed 1430 genes whose expression remained constant with respect to their expression on agar plate. These stable genes were mainly related to intracellular processes, as evidenced by the enriched GO term "metabolic process", encompassing the majority of the genes associated to biosynthetic processes. Among them, the most representative categories were amino sugar metabolism and other metabolic processes involved in amino acid, nucleic acid or ncRNA. Interestingly, some processes related to cell wall biogenesis were also constant, such as phospholipid, peptidoglycan and lipooligosaccharide biosynthetic processes, including lipid A metabolic process

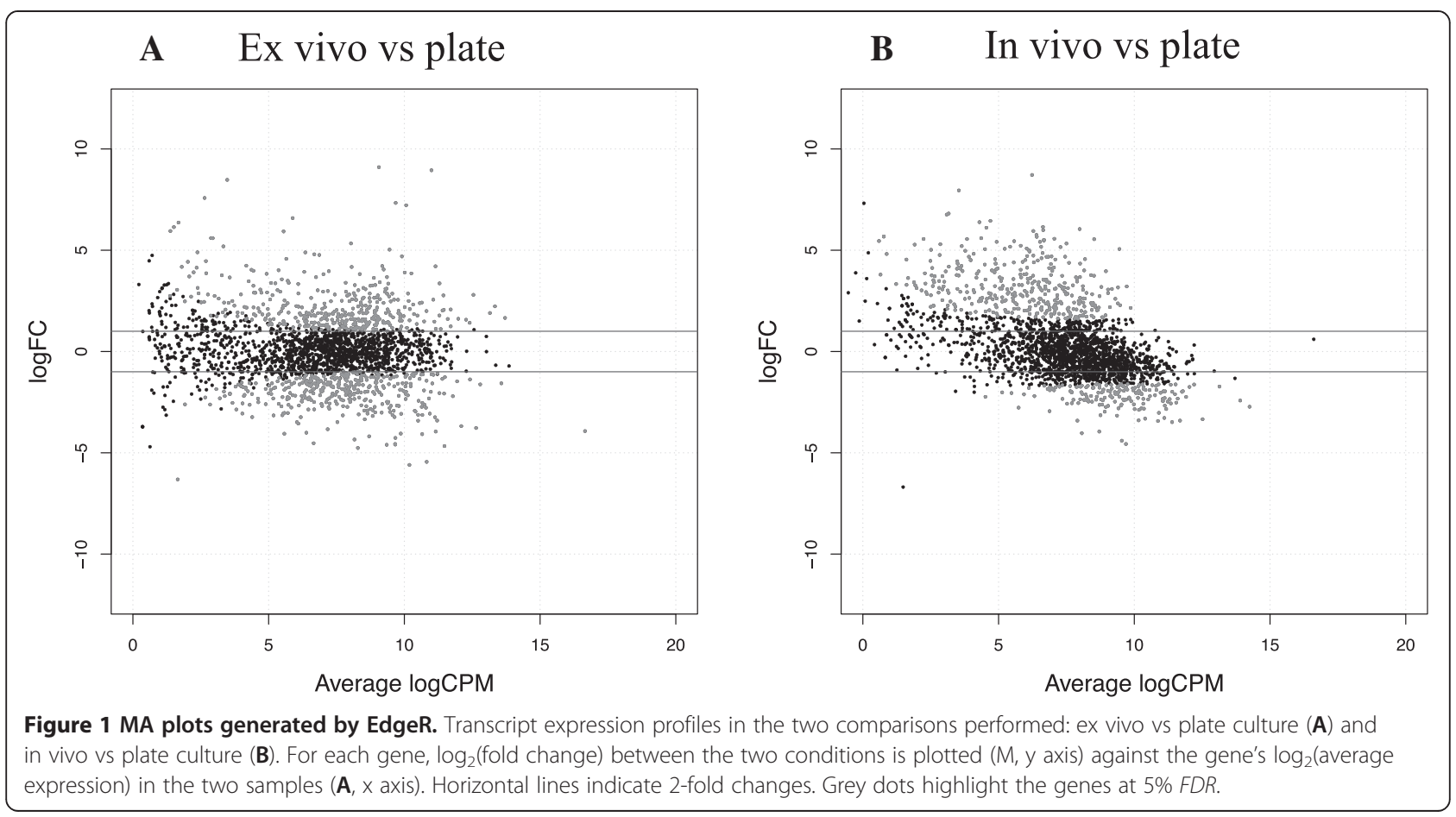


Table 1 Genes whose expression was validated using RT-qPCR with purified RNA from $H$. parasuis Nagasaki recovered after $\mathbf{2} \mathrm{h}$ incubation in lung explants (ex vivo), $\mathbf{2} \mathrm{h}$ after intratracheal infection (in vivo) and lung samples from a time-course intranasal infection ( $1 \mathrm{dpi}, 2 \mathrm{dpi}$ and $3 \mathrm{dpi}$ )

\begin{tabular}{|c|c|c|c|c|c|c|c|c|c|}
\hline \multirow[t]{3}{*}{ Locus tag } & \multirow[t]{3}{*}{ Gene type } & \multicolumn{5}{|c|}{ RT-qPCR ${ }^{1}$} & \multirow{3}{*}{ Product } & \multicolumn{2}{|l|}{ RNAseq } \\
\hline & & ex vivo & in vivo & $1 \mathrm{dpi}$ & 2 dpi & $3 \mathrm{dpi}$ & & ex vivo & in vivo \\
\hline & & & & & & & & $\log \mathrm{FC}$ & $\log \mathrm{FC}$ \\
\hline HPNK_10441 & Virulence factor & $N D^{2}$ & 4.8 & 4.2 & 3.7 & 4.6 & $\mathrm{ABC}$ transporter, permease protein & 4.2 & 4.0 \\
\hline HPNK_06205 & Virulence factor & ND & 4.9 & 4.6 & 2.9 & 4.9 & Hypothetical protein & 2.1 & 6.0 \\
\hline HPNK_09829 & Virulence factor & ND & 3.6 & 4.1 & 1.4 & 3.3 & Hypothetical protein & 5.2 & 2.8 \\
\hline HPNK_10446 & Virulence factor & ND & 3.3 & 3.5 & 2.4 & 3.1 & Hypothetical protein & 3.1 & 5.5 \\
\hline HPNK_11461 & Virulence factor & ND & 5.2 & 2.1 & 2.2 & 2.9 & Hypothetical protein & 1.8 & 3.8 \\
\hline HPNK_06565 & Virulence factor & ND & 5.4 & $H C_{T}^{3}$ & $H C_{T}$ & 3.7 & $\mathrm{Na}+/ \mathrm{H}+$ antiporter $\mathrm{NhaC}$ & 2.3 & 3.3 \\
\hline HPNK_01698 & Virulence factor & ND & 4.1 & 3.8 & 3.2 & 3.0 & VtaA2 & 1.6 & 3.1 \\
\hline HPNK_03728 & Key pathway & 2.3 & 5.0 & ND & ND & ND & 6-pyruvoyl tetrahydrobiopterin synthase & 2.6 & 1.7 \\
\hline HPNK_08953 & Key pathway & 4.3 & 2.4 & ND & ND & ND & $A B C$ transporter inner membrane subunit & 7.3 & 3.4 \\
\hline HPNK_06845 & Key pathway & 3.9 & 5.3 & ND & ND & ND & Anthranilate synthase component II & 9.1 & 1.8 \\
\hline HPNK_01064 & Key pathway & 1.3 & 5.9 & ND & ND & ND & Heme exporter protein $D$ & 1.2 & 2.0 \\
\hline HPNK_10256 & Key pathway & -2.8 & 0.6 & ND & ND & ND & PTS mannose-specific transporter subunit & -3.2 & -1.9 \\
\hline HPNK_05784 & Key pathway & -4.2 & -0.6 & ND & ND & ND & Type II citrate synthase & -4.7 & -3.4 \\
\hline HPNK_02274 & Reference & ND & - & - & - & - & BirA repressor & -0.1 & -0.1 \\
\hline HPNK_07913 & Reference & ND & - & - & - & - & MurE ligase & -0.1 & 0.1 \\
\hline HPNK_00732 & Reference & - & ND & ND & ND & ND & $50 S$ ribosomal protein $L 5$ & -0.5 & -1.7 \\
\hline HPNK_07703 & Reference & - & ND & ND & ND & ND & Translocation protein TolB & -0.2 & -0.4 \\
\hline HPNK_09689 & Reference & - & ND & ND & ND & ND & $50 S$ ribosomal protein $L 2$ & 0.7 & -0.6 \\
\hline HPNK_01728 & Reference & - & ND & ND & ND & ND & pyruvate dehydrogenase subunit E1 & 0.0 & -1.7 \\
\hline
\end{tabular}

${ }^{1}$ log2 fold changes; ${ }^{2}$ Not determined; ${ }^{3}$ the gene was amplified but the high CT values obtained were not sufficient to calculate differential expression.

(Additional file 3A). Among the up-regulated genes in ex vivo conditions, GO enrichment revealed that genes related to "transporter activity", within the "membrane" category, were enriched, as was also found in the in vivo experiment (see below). Some of these membrane-related genes were associated to pathogenesis functions (Additional file 4). Other ex vivo GO terms within the up-regulated genes were related to RNA processing, nucleic acid metabolic process, RNA metabolic process, including 21 genes related to tRNA processing (Additional file 3B). Among the most induced genes $(F D R<0.001)$ were: anthranilate synthase components I and II, glycerol-3phosphate transporter, 2 hypothetical proteins, $\mathrm{ABC}$ transporter inner membrane subunit, anthranilate phosphoribosyltransferase, phage capsid scaffolding protein, and phage phi-c31 gp35-like protein. Notably, a high induction ( $>64$ fold) of the operon related to anthranilate metabolism was observed after ex vivo incubation. Anthranilate is a key intermediate in aromatic amino acid biosynthesis and pyruvate production, and induction of its operon was also observed after in vivo infection (see below). On the contrary, the GO terms overrepresented among the set of down-regulated genes were related to intracellular pathways, with metabolism as the most represented, evidenced by the presence of GO terms related to stress response, sugar and ion transport, carbohydrate catabolism, tricarboxylic acid (TCA) cycle, nucleoside biosynthetic process, glyoxylate metabolic process and dicarboxylic acid metabolic process (Additional file 3C). Among the most repressed genes $(F D R<0.001)$ were the following: long-chain fatty acid transport protein $\mathrm{P} 1 / 47 \mathrm{kDa}$ outer membrane protein, methyl-galactoside $\mathrm{ABC}$ transporter galactose-binding periplasmic protein $\mathrm{MglB}$, cold shock-like protein $\mathrm{CspD}$, type II citrate synthase, universal stress protein A, maltose $\mathrm{ABC}$ transporter periplasmic protein, sigma 54 modulation protein and a hypothetical protein.

In an attempt to validate the ex vivo model, a short in vivo incubation was performed. Comparison of the DEG detected in both conditions showed considerable overlap; 120 genes were up-regulated in both conditions, belonging mainly to membrane-associated genes, mobile genetic elements and hypothetical proteins (Table 2 and Additional file 3D). In contrast to the stable genes, which were mainly related to intracellular metabolism, up-regulated genes after the in vivo infection consisted predominantly of the category "membrane"; with 57 genes that encoded membrane proteins, and 38 of those 
Table 2 Results of the comparison between the sets of differentially expressed genes (DEG) and enriched Gene Ontology (GO) terms between Nagasaki recovered after $2 \mathrm{~h}$ incubation in lung explants (ex vivo) or recovered $2 \mathrm{~h}$ after intratracheal infection (in vivo)

\begin{tabular}{llll}
\hline Gene group & Unique ex vivo & Shared & Unique in vivo \\
\hline DEGs UP & 273 & 120 & 249 \\
DEGs DOWN & 273 & 99 & 74 \\
GOs UP & 74 & 5 & 46 \\
GOs DOWN & 68 & 115 & 102 \\
\hline
\end{tabular}

directly tagged as transporters. In addition, 14 of the upregulated membrane genes were specific of the "outer membrane", including several virulence associated trimeric autotransporters $v t a A$. Most of these genes were also up-regulated ex vivo (Additional file 4) and included functions usually related to virulence such as nutrient transport, drug export, adhesion and mobile genetic elements including transposases and phages. Some of the genes induced in both conditions were $\mathrm{ABC}$ transporter inner membrane subunit, $\mathrm{ABC}$ transporter permease protein, $\mathrm{Na}+/ \mathrm{H}+$ antiporter, biopolymer transport ExbD protein, sn-glycerol-3-phosphate dehydrogenase subunit A, a drug/metabolite transporter (DMT) superfamily permease and several VtaA. In addition, the analysis also found genes that were induced only under one of the two conditions studied. Thus, genes uniquely induced ex vivo, although with subtle changes in vivo, included an iron-uptake permease inner membrane protein, an iron(III) ABC transporter ATP-binding protein, an oligopeptide $A B C$ transporter permease and the peptide transport periplasmic protein SapA. However, some genes were only induced in vivo, but again with subtle changes ex vivo, such as the recombination protein 2, Mu-like phage gp25, heme/hemopexin utilization protein $\mathrm{C} /$ outer membrane receptor protein, Tfp pilus assembly pathway component PilC, competence protein E and phosphatidylglycerophosphatase $\mathrm{B}$. Among the in vivo down-regulated genes, "metabolic process" was the most represented category with 125 genes, comprising both anabolic and catabolic processes. However, these genes were different from the ones found with stable expression. The most abundant metabolic categories were those related to translation, cellular carbohydrate metabolic process, TCA cycle, electron transport chain, nucleotide metabolic process, glycosyl compound biosynthetic process and monocarboxylic acid metabolic process (Additional file $3 \mathrm{E})$. Forty-two genes in the "membrane" category were also found down-regulated, including carbohydrate and ion transporters. Key indicators of low growth rate during lung infection compared to plate culture were also revealed by the down-regulation of the ATP synthase subunits, responsible for generating ATP via the electrochemical gradient, as well as glucose and manose phosphoenolpyruvatedependent sugar phosphotransferase systems (PTS). Also, 3 subunits of the $\mathrm{Na}(+)-\mathrm{NQR}$ complex were down-regulated, suggesting anaerobic growth, as previously reported for other bacterial species [35]. The $\mathrm{Na}(+)-\mathrm{NQR}$ complex plays an important role in bacterial energy metabolism [36].

To support this model of short in vivo infection, alveolar macrophages from the BALF sample were analysed. Alveolar macrophages recovered after the $2 \mathrm{~h}$ infection, showed increased expression level of CD163, SLAI and SLAII when compared to the macrophages recovered from the non-infected pig. Sialoadhesin did not show different expression in the alveolar macrophages of the infected and the non-infected piglet (Figure 2). These results indicate that this short infection was enough for the animal to detect the presence of bacteria and respond to it.

Up-regulated membrane genes and their conservation in virulent $H$. parasuis strains

The observation of numerous membrane-related genes that were up-regulated in both ex vivo and in vivo conditions was studied in more detail. Transmembrane topology and signal peptides were predicted in the putative proteome of Nagasaki using Phobius. A total of $225 \mathrm{pu}-$ tative proteins were predicted to have signal peptide, 360 to have transmembrane domains and 42 to have both signal peptide and transmembrane domains. These 627 genes are referred to as "membrane-related genes"

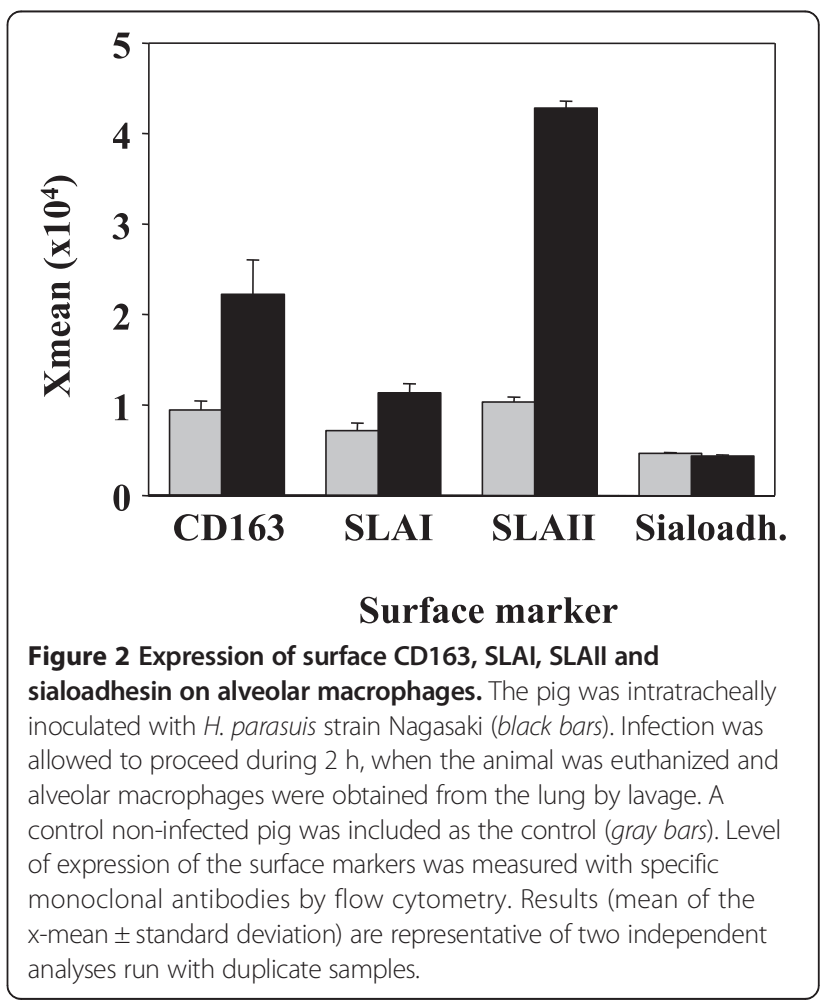


for simplicity through the rest of the text. All these membrane-related genes in the Nagasaki genome were compared to the up-regulated genes in vivo or ex vivo, confirming the high proportion observed using GO terms. A total of 115 and 108 of those 627 were upregulated ex-vivo and in-vivo, respectively. A comparison of these up-regulated membrane-related genes between ex vivo and in vivo confirmed the tendency observed with GO terms, with 36 shared genes. Next, in order to explore if these genes were unique to virulent strains, the list of up-regulated membrane-related genes was compared to a database of virulent and non-virulent genomic sequences of $H$. parasuis. We found 41 up-regulated membrane genes that were only present in virulent strains, or at most in one non-virulent strain. Twelve of these 41 genes were up-regulated in vivo and ex vivo, while 22 and 7 genes were uniquely up-regulated in vivo and ex vivo, respectively (Table 3). Shared up-regulated genes between the in vivo and ex vivo conditions included an $A B C$ transporter permease, four genes of unknown function, $\mathrm{Na}+/ \mathrm{H}+$ antiporter $n h a C$, a transposase mutator family protein and five virulence-associated autotransporters VtaAs (vtaA1, vtaA2, vtaA4, vtaA7 and vtaA11). The fact that $v t a A s$ are assembled with no gaps in the Nagasaki genome used in the analysis strengthens the value of these results. The 22 genes uniquely up-regulated under in vivo conditions, included bacteriophage tail protein GPT, nine hypothetical proteins, three ISAs1 family transposases, putative iron compound $\mathrm{ABC}$ transporter permease, putative phage DNA replication protein $\mathrm{O}$, two pyruvate kinases, two Sel1 domain repeat-containing proteins, and three vtaAs (vtaA3,vtaAs and vtaA9). On the other hand, the 7 genes uniquely up-regulated in the ex vivo experiment included those encoding a CDPdiglyceride pyrophosphorylase, copper-transporting P-type ATPase, exonuclease III, two hypothetical proteins, phagelike minor tail protein and polysaccharide biosynthesis protein CapD (Table 3). Due to the essential role of pyruvate kinase enzyme in glycolysis, lack of this gene in non-virulent strains would be unlikely. The two genes annotated as pyruvate kinases and found only in virulent strains were HPNK_07393 and HPNK_09849. Further InterPro inspection results revealed that none of these two genes really belongs to the pyruvate kinase family (IPR001697), their protein annotation thus being incorrect. Although these two genes had a positive prediction for signal peptide, InterPro functional annotation was scarce. Their predicted domains are not specific to a particular pathway: a tetratricopeptide-like helical domain (IPR011990) for HPNK_07393 and a polyketide cyclase SnoaL-like domain (IPR009959) for HPNK_09849. In fact, there are at least three more genes annotated as "pyruvate kinase" in H. parasuis Nagasaki: HPNK_06225, HPNK_05314 and HPNK_07388. Only HPNK_06225 is specific to virulent strains, having a polyketide cyclase SnoaL-like domain (IPR009959). The other two, HPNK_ 05314, the largest one, seems to be the true pyruvate kinase (IPR001697), while HPNK_07388, again with a wrong annotation, has no predicted domains.

Expression of four genes of unknown function, the $v t a A 2$, the $\mathrm{Na}+/ \mathrm{H}+$ antiporter $n h a C$ and an $\mathrm{ABC}$ transporter permease protein was validated via RT-qPCR in the in vivo sample (Table 1). These genes were selected because they were found up-regulated in both in vivo and ex vivo conditions and were only detected in virulent strains. The up-regulation was confirmed for all seven genes, and positive correlation was found between RT-qPCR and RNA-seq $(r=0.8, P<0.05)$. An increase in RT-qPCR fold change was correlated with an increase in RNA-seq fold change. In addition, the transcription of these genes was examined by RT-qPCR in BALF samples from a previous experimental infection with the Nagasaki strain. These genes were found to be up-regulated in bacteria recovered at 1,2 and 3 dpi from the lungs of piglets intranasally inoculated with Nagasaki, with the exception of the $\mathrm{Na}+\mathrm{H}+$ antiporter $n h a C$, whose expression was too low for evaluation by this method.

\section{ncRNA in $H$. parasuis and their differential expression}

Antisense expression was observed for all six sequenced samples, and was processed for further investigation. Count files from the counting step were used [GEO: GSE63851]. Determination of the sense/antisense expression ratio was performed. Due to the presence of some extreme values for antisense transcripts, median values were used. For ex vivo samples, sense to antisense ratios varied between 13 and 19, while a ratio of 43-68 was observed for their corresponding plate samples; thus evidencing a clear change in the sense to antisense ratio during ex vivo incubation. This tendency was also observed for the in vivo sample, where sense to antisense ratios of 4 and 15 were obtained for in vivo and its corresponding plate sample, respectively. These observations suggest that proportions were more equilibrated in the in vivo and ex vivo samples, while plate samples were biased towards sense expression. Normality of sense and antisense counts was assessed using the Shapiro-Wilk test. In all 6 cases $P$ values were below 0.05 indicating non-normality. Therefore, correlations were assessed using the non-parametric Spearman rank-correlation test. The results indicate that in vivo sense and antisense expression was positively correlated $(r=0.43, P<0.05)$. Similarly, plate sense and antisense expression was also positively correlated $(r=0.66, P<0.05)$. However, this tendency was not observed for the ex vivo and their corresponding plate samples $(r=0.07-0.15, P<0.05)$. All calculations were carried out using $\mathrm{R}$ statistical software [37]. Since a negative correlation would have been 
Table 3 List of up-regulated membrane genes $(F D R<0.05)$ unique of virulent $H$. parasuis strains $(\mathrm{V})$ or present at most in one non-virulent strain (one NV)

\begin{tabular}{|c|c|c|c|}
\hline Locus & in vivo/ex vivo ${ }^{1}$ & Product & Unique V/one NV \\
\hline HPNK_10441 & in vivo/ex vivo & ABC transporter, permease protein & Unique $\mathrm{V}$ \\
\hline HPNK_03268 & in vivo & Bacteriophage tail protein GPT & Unique $\mathrm{V}$ \\
\hline HPNK_04602 & ex vivo & CDP-diglyceride pyrophosphorylase & Unique $\mathrm{V}$ \\
\hline HPNK_01144 & ex vivo & Copper-transporting P-type ATPase & Unique $\mathrm{V}$ \\
\hline HPNK_07008 & ex vivo & Exonuclease III & Unique $\mathrm{V}$ \\
\hline HPNK_00812 & in vivo/ex vivo & Hypothetical protein & One NV \\
\hline HPNK_06205 & in vivo/ex vivo & Hypothetical protein & Unique $\mathrm{V}$ \\
\hline HPNK_09829 & in vivo/ex vivo & Hypothetical protein & Unique $\mathrm{V}$ \\
\hline HPNK_10446 & in vivo/ex vivo & Hypothetical protein & Unique $\mathrm{V}$ \\
\hline HPNK_09189 & ex vivo & Hypothetical protein & One NV \\
\hline HPNK_00632 & ex vivo & Hypothetical protein & Unique $\mathrm{V}$ \\
\hline HPNK_00572 & in vivo & Hypothetical protein & Unique $\mathrm{V}$ \\
\hline HPNK_05179 & in vivo & Hypothetical protein & Unique $\mathrm{V}$ \\
\hline HPNK_00277 & in vivo & Hypothetical protein & Unique $\mathrm{V}$ \\
\hline HPNK_08083 & in vivo & Hypothetical protein & One NV \\
\hline HPNK_10351 & in vivo & Hypothetical protein & Unique V \\
\hline HPNK_00312 & in vivo & Hypothetical protein & One NV \\
\hline HPNK_11566 & in vivo & Hypothetical protein & One NV \\
\hline HPNK_11461 & in vivo & Hypothetical protein & One NV \\
\hline HPNK_04137 & in vivo & Hypothetical protein & One NV \\
\hline HPNK_04889 & in vivo & ISAs1 family transposase & One NV \\
\hline HPNK_00080 & in vivo & ISAs1 family transposase & One NV \\
\hline HPNK_04984 & in vivo & ISAs1 family transposase & One NV \\
\hline HPNK_06565 & in vivo/ex vivo & $\mathrm{Na}+/ \mathrm{H}+$ antiporter $\mathrm{NhaC}$ & Unique $\mathrm{V}$ \\
\hline HPNK_11451 & ex vivo & Phage-like minor tail protein & One NV \\
\hline HPNK_07568 & ex vivo & Polysaccharide biosynthesis protein CapD & Unique V \\
\hline HPNK_05239 & in vivo & putative iron compound $A B C$ transporter permease & Unique $\mathrm{V}$ \\
\hline HPNK_00090 & in vivo & Putative phage DNA replication protein $\mathrm{O}$ & One NV \\
\hline HPNK_09849 & in vivo & Polyketide cyclase SnoaL-like domain protein & Unique $\mathrm{V}$ \\
\hline HPNK_07393 & in vivo & Tetratricopeptide-like helical domain protein & Unique $\mathrm{V}$ \\
\hline HPNK_07408 & in vivo & Sel1 domain protein, repeat-containing protein & Unique $\mathrm{V}$ \\
\hline HPNK_07403 & in vivo & Sel1 domain protein, repeat-containing protein & Unique $\mathrm{V}$ \\
\hline HPNK_00210 & in vivo/ex vivo & Transposase, Mutator family protein & One NV \\
\hline HPNK_09439 & in vivo/ex vivo & VtaA1 & Unique $\mathrm{V}$ \\
\hline HPNK_01479 & in vivo/ex vivo & VtaA11 & Unique V \\
\hline HPNK_01698 & in vivo/ex vivo & VtaA2 & Unique $\mathrm{V}$ \\
\hline HPNK_07258 & in vivo & VtaA3 & Unique $\mathrm{V}$ \\
\hline HPNK_10146 & in vivo/ex vivo & VtaA4 & Unique $\mathrm{V}$ \\
\hline HPNK_02582 & in vivo/ex vivo & VtaA7 & Unique V \\
\hline HPNK_01967 & in vivo & VtaA8 & Unique V \\
\hline HPNK_10812 & in vivo & VtaA9 & Unique $\mathrm{V}$ \\
\hline
\end{tabular}

${ }^{1}$ up-regulated in vivo, ex vivo or in both samples (in vivo/ex vivo). 
expected by reducing sense expression via sense/asRNA pairing, these unexpected results suggest that no sense/ antisense regulation is occurring and that there are other factors influencing an increase in antisense expression. Pervasive transcription has been reported both for prokaryotes and mammals [38,39], but it has not yet been described for $H$. parasuis. To validate some of the observed asRNA, RT-PCR was performed. Four asRNA were validated both in the in vivo and plate culture samples. These asRNA overlapped with genes annotated as 6-pyruvoyl tetrahydrobiopterin synthase, type II citrate synthase, copper-transporting P-type ATPase and anthranilate synthase component II (Figure 3A and
Additional file 1). The positive correlation sense-asRNA observed for the in vivo sample was studied in more detail. Antisense differential expression was calculated, finding a considerable number of differentially expressed asRNA $(F D R<0.05), 213$ and 268 up- and down-regulated, respectively (Additional file $2 \mathrm{C}$ ). GO enrichments were also performed for the set of differentially expressed asRNA (Additional files 3F and G) and were compared to sense enrichment results. We found an important overlap between the enriched GO terms of the repressed asRNA and sense RNA, with 135 matches (Figure 3B). These pathways were mainly related to intracellular genes, mostly metabolism. Among them,

A
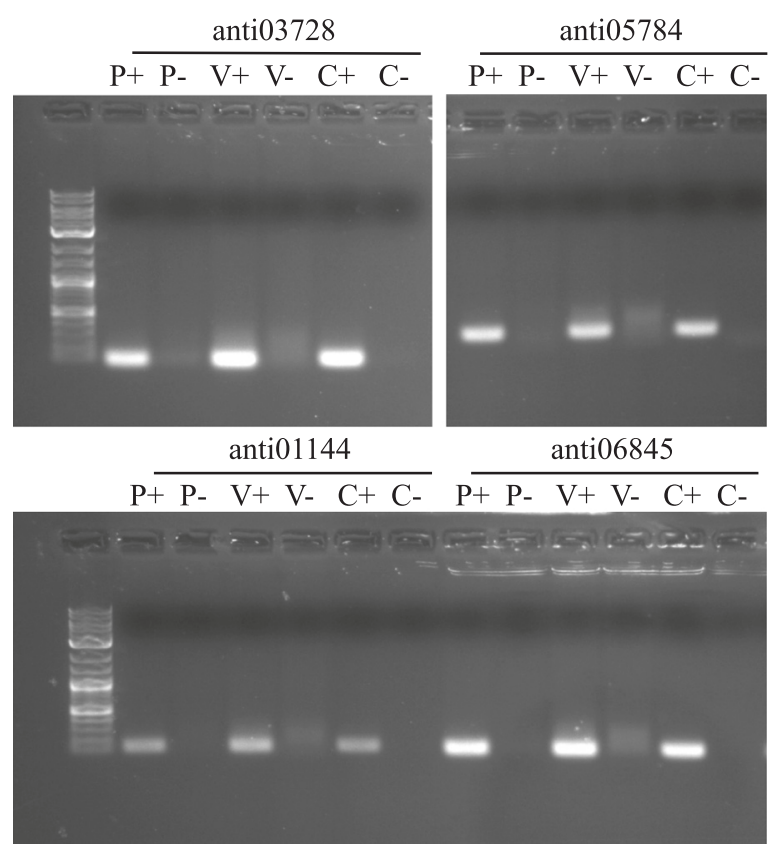

$\mathrm{B}$

B asRNA DOWN sense RNA UP

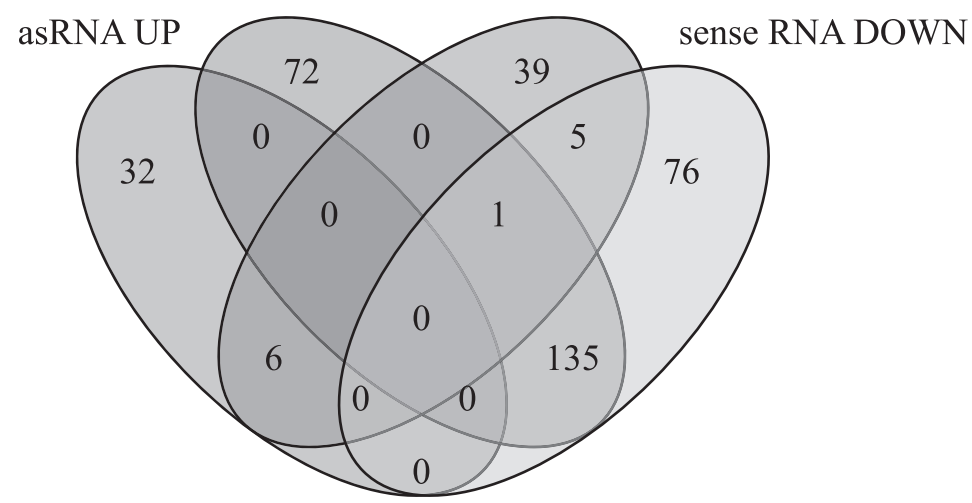

Figure 3 Validation of antisense RNA (asRNA) expressed in vivo and in plate culture. A Electrophoresis gel of four asRNA amplified by RT-PCR showing amplification results for RNA purified from plate culture with RT step (P+), plate culture without RT step (P-), 2 h in vivo sample with RT $(\mathrm{V}+)$ or without RT step (V-). Genomic DNA was included as the positive control $(\mathrm{C}+)$ and water as the negative control (C-). B Venn diagram showing overlapping Gene Ontology (GO) enriched terms for up- and down-regulated asRNA or sense RNA at 5\% FDR. 
carbohydrate catabolic process, phosphoenolpyruvatedependent sugar phosphotransferase system, translation, tricarboxylic acid cycle, glycosyl compound biosynthetic process, purine ribonucleoside triphosphate biosynthetic process and monocarboxylic acid metabolic process were the main representatives. These results confirm that the positive correlation of sense and antisense expression was also linked to some common pathways. Some overlap was also observed between the pathways of the induced asRNA and sense RNA, with 6 shared GO terms including pathogenesis and pilus organization.

\section{Discussion}

After an initial colonization of the upper respiratory tract, lung infection is one of the early steps in H. parasuis pathogenesis. Therefore, a deep understanding of the factors involved in survival of this bacterium in the lung will be useful for disease control. In this study, we have shown that the Nagasaki strain of $H$. parasuis modulates its gene expression during in vivo lung infection, but also in an ex vivo lung infection model. Nutrient acquisition, expression of genes related to pathogenesis and a reduced metabolism are the main signatures of $H$. parasuis adaptation to the lung environment. Some of the up-regulated genes during lung infection were only found in the genomes of virulent strains, indicating their potential as virulence factors. In addition, previously reported $H$. parasuis virulence factors were also upregulated in the lung.

One of the most important limitations to elucidate bacterial gene expression during host colonisation is the low RNA quality that is usually obtained from field or experimental infections. Moreover, the proportionally low quantity of mRNA relative to rRNA makes transcriptomics a challenging strategy. In our case, we were not able to obtain enough RNA of good quality for highthroughput sequencing from samples taken at 1 to 4 days post-inoculation from an infection previously performed by us $[14,27]$. To overcome these limitations, we decided to use a short in vivo infection and an ex vivo lung infection model. Processing of BALF samples for RNA purification coupled with additional enrichment steps such as differential centrifugation to remove host cells and removal of host and bacterial rRNA resulted in a modest but sufficient RNA quantity to study bacterial gene expression by metatranscriptomics. In our study, $3 \mathrm{M}$ reads from the ex vivo sample and $18 \mathrm{M}$ reads from the in vivo sample were enough to study the gene expression of $95 \%$ of the genes. These sequencing depths were sufficient to reach the coverage recommended to quantify bacterial gene expression [40].

In vivo incubation revealed numerous genes that were differentially expressed. Even prior to pathway analysis, visualization of the MA differential expression plot showed a particular "wave" shape, thus pointing to up-regulation of genes normally not expressed during laboratory agar plate growth, and, conversely, down-regulation of the most expressed ones. Increased nutrient capture, together with metabolism repression were the main adaptive responses of $H$. parasuis in the lung. Interestingly, a similar picture has been observed in other in vivo transcriptome studies of other bacterial species from the Pasteurellaceae family, such as Mannheimia hemolytica, responsible for bovine pneumonic pasteurellosis [17] or for Actinobacillus pleuropneumoniae, the etiological agent of porcine pleuropneumonia [18], thus suggesting slower metabolism and replication rate in vivo compared to growth in a rich culture media. Deslandes et al. [18] also found that the "Transport and Binding Protein" functional class was overrepresented among the up-regulated genes in A. pleuropneumoniae under in vivo conditions. Here, we found that the most represented cellular component among the up-regulated genes in the lung was the one related to membrane proteins. Some of these genes were distributed in different categories of nutrient capture, such as sugar and amino acid transport. These findings indicate that "nutritional virulence", previously reported for other bacterial pathogens [41], could be an important survival strategy for $H$. parasuis during lung colonization. Moreover, some of these genes were involved in iron, nitrate and sulfonate acquisition, which are vital for energy generation and have been previously reported to be upregulated in $H$. parasuis grown under iron-restriction in vitro [42]. Furthermore, up-regulation of cytochrome biogenesis, heme transport and some components of the electron transport chain add additional value to the hypothesis that maintenance of anabolic/catabolic balance could be important for survival in the host. Additionally, $A B C$ transporters, which have been found to be upregulated in the current study, have been reported to be essential elements for bacterial survival in the host $[43,44]$. Interestingly, some $\mathrm{ABC}$ transporters could have been acquired via horizontal gene transfer in $H$. parasuis [23]. While $A B C$ transporters were found up-regulated, the PTS sugar transport systems were down-regulated, indicating that different sugar transporters could be used depending on the environmental conditions, by opposite regulation of these two ATP-dependent transporters [45].

One of the main questions we tried to answer in this work was if any of the previously reported virulence factors were up-regulated in vivo. Genes involved in surface polysaccharides, lipid A or proteases did not show an overall increased expression. However, iron, hemin, hemopexin or transferring binding proteins showed a tendency for up-regulation. Also, mining for up-regulated membranerelated genes that were unique to virulent strains revealed 42 putative virulence factors. Several of these gene categories have been previously linked to virulence in bacteria, 
such as iron acquisition or $A B C$ transporters [46], frequently subjected to horizontal genetic transfer via mobile genetic elements [47]. On the contrary, some proteins previously reported as putative virulence factors in $\mathrm{H}$. parasuis, such as outer membrane protein P2 (OmpP2), cytolethal distending toxins, heptosyltransferases, which have been involved in adhesion, invasion or serum resistance [48-50], showed subtle down-regulation. This down-regulation could indicate that these molecules do not play a specific role in lung survival. Finally, vtas were up-regulated in vivo, including $v t a A 8$ and $v t a A 9$, which have been demonstrated to play a role in phagocytosis resistance in $H$. parasuis [15]. Due to the complex structure of these genes, with repetitions, common domains and variability of the passenger domain [51], careful analysis should be performed to determine their presence in different strains. Previous knowledge about these genes indicates that $v t a A 1$ to $v t a A 11$ are associated with virulence [52]. We should highlight that a total of 128 up-regulated genes are currently annotated as genes of unknown function. Some of these genes of unknown function were detected by Phobius as membrane-related and were only found in virulent strains. These genes of unknown function require further research, since they could play a role in novel pathogenic strategies [53]. Additionally, the validation of up-regulation of 4 of these genes of unknown function in other in vivo samples supports the role of these membrane proteins during infection as well as their use as vaccine candidates.

Development of ex vivo models has been reported as useful to mimic in vivo conditions [54]. Currently, an ex vivo infection model is absent for $H$. parasuis. In this work we tried to bypass the current limitations of in vivo experiments by developing an ex vivo lung infection model. A $2 \mathrm{~h}$ ex vivo incubation resulted in high yield of bacterial RNA recovery. Importantly, DEG were detected in similar pathways as in the in vivo sample, validating this strategy as a good alternative for $H$. parasuis host-pathogen interaction studies at the respiratory tract. However, the comparison of the results from the ex vivo and in vivo infections also found differences between these samples, indicating that the true expression of some genes during infection can only be revealed in vivo. The advent of ultra-high throughput sequencing would allow previously unimagined sequencing depths, which will be useful in the analysis of samples from in vivo experiments. For instance, analysis of other sample types containing a lower number of bacterial cells, such as samples from later stages of infection, will open new possibilities to study the progression of disease in the lung.

One of the key factors to develop an effective vaccine to control a bacterial infection is to know its pathogenic lifestyle. We found that $H$. parasuis changes its global gene expression during lung infection, with an overall tendency to up-regulate membrane-related genes involved in carbon acquisition, iron binding and pathogenesis. A strong down-regulation of metabolism was observed, however, suggesting an importance of a lower replicative rate as an adaptation for host survival. These metabolic adaptations were accompanied by induction of well-known virulence factors as well as other genes that, although less explored, could be behind novel virulence mechanisms. In summary, this work serves as a useful infection model for $H$. parasuis, adding value to some up-regulated virulence factors for their use as vaccine candidates.

\section{Additional files}

\section{Additional file 1: Primers. Primers used to validate expression pattern} of RNA-seq data.

Additional file 2: Differential expression of Nagasaki genes.

Differential expression of genes ex vivo versus plate culture (A), in vivo versus plate culture (B) and antisense RNA in vivo versus plate culture (C).

Additional file 3: GO enrichments. Gene set enrichments for housekeeping, up-regulated and down-regulated genes. Enriched GO categories among the up- and down-regulated antisense RNA in vivo are also shown.

Additional file 4: Membrane genes. Membrane genes up-regulated in vivo, ex vivo or in both conditions. Differential expression is also shown.

\section{Competing interests}

The authors declare that they have no competing interests.

\section{Authors' contributions}

BBO carried out the RNA sample processing, bioinformatic analysis, RT-qPCRs and drafted the manuscript. VA studied the expression of surface markers of PAM, conceived the study and helped to draft the manuscript. KJH, AWT and DJM provided the $H$. parasuis genomic sequences and revised the manuscript. All authors read and approved the final manuscript.

\section{Acknowledgments}

This work was supported by grant AGL2010-15232 from the Ministerio de Economía y Competitividad of Spain to VA. BBO is a recipient of a FPI fellowship from the Spanish Ministerio de Economía y Competitividad. The authors would like to acknowledge Joaquim Segales for helping in the animal infection and Núria Galofré for laboratory processing of infected pig lungs.

\section{Author details}

${ }^{1}$ Centre de Recerca en Sanitat Animal (CReSA), Institut de Recerca i Tecnologia Agroalimentàries (IRTA), Campus de la Universitat Autònoma de Barcelona, Bellaterra, 08193 Cerdanyola del Vallès, Spain. ²Department of Veterinary Medicine, University of Cambridge, Madingley Road, Cambridge CB3 OES, UK

Received: 2 February 2015 Accepted: 21 July 2015

Published online: 23 September 2015

\section{References}

1. Aragon V, Segalés J, Oliveira S (2012) Glässer's disease. In: Karriker LA Ramirez A, Schwartz KJ, Stevenson GW (eds) Edited by Zimmerman JJ. Diseases of swine. Wiley-Blackwell publishing, lowa

2. San Millan A, Escudero JA, Catalan A, Nieto S, Farelo F, Gibert M, Moreno MA, Dominguez L, Gonzalez-Zorn B (2007) Beta-lactam resistance in Haemophilus parasuis is mediated by plasmid pB1000 bearing blaROB-1. Antimicrob Agents Chemother 51:2260-2264

3. Zhang Q, Liu J, Yan S, Yang Y, Zhang A, Jin M (2013) Fluoroquinoloneresistant Haemophilus parasuis isolates exhibit more putative virulence factors than their susceptible counterparts. J Clin Microbiol 51:3130-3131 
4. Chen LP, Cai XW, Wang XR, Zhou XL, Wu DF, Xu XJ, Chen HC (2010) Characterization of plasmid-mediated lincosamide resistance in a field isolate of Haemophilus parasuis. J Antimicrob Chemother 65:2256-2258

5. Nedbalcova K, Kucerova Z, Krejci J, Tesarik R, Gopfert E, Kummer V, Leva L, Kudlackova H, Ondriasova R, Faldyna M (2011) Passive immunisation of post-weaned piglets using hyperimmune serum against experimental Haemophilus parasuis infection. Res Vet Sci 91:225-229

6. Olvera A, Ballester M, Nofrarias M, Sibila M, Aragon V (2009) Differences in phagocytosis susceptibility in Haemophilus parasuis strains. Vet Res 40:24

7. Cheng G, Hao H, Xie S, Wang X, Dai M, Huang L, Yuan Z (2014) Antibiotic alternatives: the substitution of antibiotics in animal husbandry? Front Microbiol 5:217

8. Vouloumanou EK, Makris GC, Karageorgopoulos DE, Falagas ME (2009) Probiotics for the prevention of respiratory tract infections: a systematic review. Int J Antimicrob Agents 34:197.e1-10

9. Costa-Hurtado M, Aragon V (2013) Advances in the quest for virulence factors of Haemophilus parasuis. Vet J 198:571-576

10. Zhang B, Tang C, Liao M, Yue H (2014) Update on the pathogenesis of Haemophilus parasuis infection and virulence factors. Vet Microbiol 168:1-7

11. Ruter C, Hardwidge PR (2014) 'Drugs from bugs': bacterial effector proteins as promising biological (immune-) therapeutics. FEMS Microbiol Lett 351:126-132

12. Eisenreich W, Dandekar T, Heesemann J, Goebel W (2010) Carbon metabolism of intracellular bacterial pathogens and possible links to virulence. Nat Rev Microbiol 8:401-412

13. Vahle JL, Haynes JS, Andrews JJ (1997) Interaction of Haemophilus parasuis with nasal and tracheal mucosa following intranasal inoculation of cesarean derived colostrum deprived (CDCD) swine. Can J Vet Res 61:200-206

14. Bello-Orti B, Costa-Hurtado M, Martinez-Moliner V, Segales J, Aragon V (2014) Time course Haemophilus parasuis infection reveals pathological differences between virulent and non-virulent strains in the respiratory tract. Vet Microbiol 170:430-437

15. Costa-Hurtado M, Ballester M, Galofre-Mila N, Darji A, Aragon V (2012) VtaA8 and VtaA9 from Haemophilus parasuis delay phagocytosis by alveolar macrophages. Vet Res 43:57

16. Jorth P, Trivedi U, Rumbaugh K, Whiteley M (2013) Probing bacterial metabolism during infection using high-resolution transcriptomics. J Bacteriol 195:4991-4998

17. Sathiamoorthy S, Hodgins DC, Shewen PE, Highlander SK, Lo RY (2011) A snap-shot of Mannheimia hemolytica A1 gene expression during infection in the bovine host. FEMS Microbiol Lett 325:148-154

18. Deslandes V, Denicourt M, Girard C, Harel J, Nash JH, Jacques M (2010) Transcriptional profiling of Actinobacillus pleuropneumoniae during the acute phase of a natural infection in pigs. BMC Genomics 11:98

19. Boyce JD, Wilkie I, Harper M, Paustian ML, Kapur V, Adler B (2002) Genomic scale analysis of Pasteurella multocida gene expression during growth within the natural chicken host. Infect Immun 70:6871-6879

20. Kim S, Park J, Lee J, Shin D, Park DS, Lim JS, Choi IY, Seo YS (2014) Understanding pathogenic Burkholderia glumae metabolic and signaling pathways within rice tissues through in vivo transcriptome analyses. Gene 547:77-85

21. Son MS, Matthews WJ Jr, Kang Y, Nguyen DT, Hoang TT (2007) In vivo evidence of Pseudomonas aeruginosa nutrient acquisition and pathogenesis in the lungs of cystic fibrosis patients. Infect Immun 75:5313-5324

22. Camejo A, Buchrieser C, Couve E, Carvalho F, Reis O, Ferreira P, Sousa S, Cossart P, Cabanes D (2009) In vivo transcriptional profiling of Listeria monocytogenes and mutagenesis identify new virulence factors involved in infection. PLoS Pathog 5:e1000449

23. Bello-Orti B, Aragon V, Pina-Pedrero S, Bensaid A (2014) Genome comparison of three serovar 5 pathogenic strains of Haemophilus parasuis: insights into an evolving swine pathogen. Microbiology 160:1974-1984

24. Markowitz VM, Chen IM, Palaniappan K, Chu K, Szeto E, Grechkin Y, Ratner A, Jacob B, Huang J, Williams P, Huntemann M, Anderson I, Mavromatis K, Ivanova NN, Kyrpides NC (2012) IMG: the integrated microbial genomes database and comparative analysis system. Nucleic Acids Res 40:D115-D122

25. Caspi $\mathrm{R}$, Altman $\mathrm{T}$, Billington $\mathrm{R}$, Dreher $\mathrm{K}$, Foerster $\mathrm{H}$, Fulcher CA, Holland TA, Keseler IM, Kothari A, Kubo A, Krummenacker M, Latendresse M, Mueller LA, Ong Q, Paley S, Subhraveti P, Weaver DS, Weerasinghe D, Zhang P, Karp PD (2014) The MetaCyc database of metabolic pathways and enzymes and the BioCyc collection of Pathway/Genome Databases. Nucleic Acids Res 42:D459-D471
26. Bello-Orti B, Deslandes V, Tremblay YD, Labrie J, Howell KJ, Tucker AW, Maskell DJ, Aragon V, Jacques M (2014) Biofilm formation by virulent and non-virulent strains of Haemophilus parasuis. Vet Res 45:104

27. Costa-Hurtado M, Olvera A, Martinez-Moliner V, Galofre-Mila N, Martinez P, Dominguez J, Aragon V (2013) Changes in macrophage phenotype after infection of pigs with Haemophilus parasuis strains with different levels of virulence. Infect Immun 81:2327-2333

28. Anders S, McCarthy DJ, Chen Y, Okoniewski M, Smyth GK, Huber W, Robinson MD (2013) Count-based differential expression analysis of RNA sequencing data using $R$ and Bioconductor. Nat Protoc 8:1765-1786

29. Li H, Durbin R (2010) Fast and accurate long-read alignment with Burrows-Wheeler transform. Bioinformatics 26:589-595

30. Lassmann T, Hayashizaki Y, Daub CO (2011) SAMStat: monitoring biases in next generation sequencing data. Bioinformatics 27:130-131

31. Conesa A, Gotz S, Garcia-Gomez JM, Terol J, Talon M, Robles M (2005) Blast2GO: a universal tool for annotation, visualization and analysis in functional genomics research. Bioinformatics 21:3674-3676

32. Supek F, Bosnjak M, Skunca N, Smuc T (2011) REVIGO summarizes and visualizes long lists of gene ontology terms. PLos One 6:e21800

33. Kall L, Krogh A, Sonnhammer EL (2004) A combined transmembrane topology and signal peptide prediction method. J Mol Biol 338:1027-1036

34. Howell KJ, Weinert LA, Chaudhuri RR, Luan S, Peters SE, Corander J, Harris D, Angen O, Aragon V, Bensaid A, Williamson SM, Parkhill J, Langford PR, Rycroft AN, Wren BW, Holden MT, Tucker AW, Maskell DJ (2014) The use of genome wide association methods to investigate pathogenicity, population structure and serovar in Haemophilus parasuis. BMC Genomics 15:1179

35. Fadeeva MS, Yakovtseva EA, Belevich GA, Bertsova YV, Bogachev AV (2007) Regulation of expression of $\mathrm{Na}+$-translocating $\mathrm{NADH}$ :quinone oxidoreductase genes in Vibrio harveyi and Klebsiella pneumoniae. Arch Microbiol 188:341-348

36. Juarez O, Morgan JE, Nilges MJ, Barquera B (2010) Energy transducing redox steps of the $\mathrm{Na}+-$ pumping $\mathrm{NADH}$ :quinone oxidoreductase from Vibrio cholerae. Proc Natl Acad Sci U S A 107:12505-12510

37. R Development Core Team: R (2013) A language and environment for statistical computing. R Foundation for Statistical Computing, Vienna, Austria. ISBN 3-900051-07-0, URL http://www.R-project.org, 2013

38. Wade JT, Grainger DC (2014) Pervasive transcription: illuminating the dark matter of bacterial transcriptomes. Nat Rev Microbiol 12:647-653

39. Clark MB, Amaral PP, Schlesinger FJ, Dinger ME, Taft RJ, Rinn JL, Ponting CP, Stadler PF, Morris KV, Morillon A, Rozowsky JS, Gerstein MB, Wahlestedt C, Hayashizaki Y, Carninci P, Gingeras TR, Mattick JS (2011) The reality of pervasive transcription. PLoS Biol 9:e1000625

40. Haas BJ, Chin M, Nusbaum C, Birren BW, Livny J (2012) How deep is deep enough for RNA-Seq profiling of bacterial transcriptomes? BMC Genomics 13:734

41. Abu Kwaik Y, Bumann D (2013) Microbial quest for food in vivo: 'nutritional virulence' as an emerging paradigm. Cell Microbiol 15:882-890

42. Xie Q, Jin H, Luo R, Wan Y, Chu J, Zhou H, Shi B, Chen H, Zhou R (2009) Transcriptional responses of Haemophilus parasuis to iron-restriction stress in vitro. Biometals 22:907-916

43. Mazzariol A, Tokue Y, Kanegawa TM, Cornaglia G, Nikaido H (2000) High-level fluoroquinolone-resistant clinical isolates of Escherichia coli overproduce multidrug efflux protein AcrA. Antimicrob Agents Chemother 44:3441-3443

44. Martinez JL, Sanchez MB, Martinez-Solano L, Hernandez A, Garmendia L Fajardo A, Alvarez-Ortega C (2009) Functional role of bacterial multidrug efflux pumps in microbial natural ecosystems. FEMS Microbiol Rev 33:430-449

45. Untiet V, Karunakaran R, Kramer M, Poole P, Priefer U, Prell J (2013) ABC transport is inactivated by the PTS(Ntr) under potassium limitation in Rhizobium leguminosarum 3841. PLoS One 8:e64682

46. Rodriguez GM, Smith I (2006) Identification of an ABC transporter required for iron acquisition and virulence in Mycobacterium tuberculosis. J Bacteriol 188:424-430

47. Frost LS, Leplae R, Summers AO, Toussaint A (2005) Mobile genetic elements: the agents of open source evolution. Nat Rev Microbiol 3:722-732

48. Xu C, Zhang L, Zhang B, Feng S, Zhou S, Li J, Zou Y, Liao M (2013) Involvement of lipooligosaccharide heptose residues of Haemophilus parasuis SC096 strain in serum resistance, adhesion and invasion. Vet J 195:200-204

49. Zou Y, Feng S, Xu C, Zhang B, Zhou S, Zhang L, He X, Li J, Yang Z, Liao M (2013) The role of galU and galE of Haemophilus parasuis SC096 in serum resistance and biofilm formation. Vet Microbiol 162:278-284 
50. Zhang B, He Y, Xu C, Xu L, Feng S, Liao M, Ren T (2012) Cytolethal distending toxin (CDT) of the Haemophilus parasuis SC096 strain contributes to serum resistance and adherence to and invasion of PK-15 and PUVEC cells. Vet Microbiol 157:237-242

51. Pina S, Olvera A, Barcelo A, Bensaid A (2009) Trimeric autotransporters of Haemophilus parasuis: generation of an extensive passenger domain repertoire specific for pathogenic strains. J Bacteriol 191:576-587

52. Olvera A, Pina S, Macedo N, Oliveira S, Aragon V, Bensaid A (2012) Identification of potentially virulent strains of Haemophilus parasuis using a multiplex PCR for virulence-associated autotransporters (vtaA). Vet J 191:213-218

53. Shahbaaz M, Hassan Ml, Ahmad F (2013) Functional annotation of conserved hypothetical proteins from Haemophilus influenzae Rd KW20. PLoS One 8:e84263

54. Harrison F, Muruli A, Higgins S, Diggle SP (2014) Development of an ex vivo porcine lung model for studying growth, virulence, and signaling of Pseudomonas aeruginosa. Infect Immun 82:3312-3323

\section{Submit your next manuscript to BioMed Central and take full advantage of:}

- Convenient online submission

- Thorough peer review

- No space constraints or color figure charges

- Immediate publication on acceptance

- Inclusion in PubMed, CAS, Scopus and Google Scholar

- Research which is freely available for redistribution 